\title{
Online Detection and Study of Cracks in Grinding Hardening
}

\author{
Liang Ping ${ }^{1}$, Cheng Wei ${ }^{1}$, Zhao Yulong ${ }^{1}$ \\ ${ }^{1}$ Huaiyin Institute of Technology, Huai'an 223003, China
}

\begin{abstract}
Grind-Hardening technology is a new technology for cutting that combines grinding theory with quenching theory, and the heat produced during the grinding process has a great effect on the surface quality of the parts. This paper take advantage of method of acoustic emission testing to test the grinding hardened parts by online or scene. The wavelet packet is used to denoise the signal, then the denoising signal is analyzed to judge those grinding cracks or defects that may appear on the surface of the parts, and besides, this method is easy to do.
\end{abstract}

\section{Introduction}

Grind-Hardening is a new technology of cutting that combines grinding theory with quenching theory, and the technology is a integration of grinding process and surface quenching, which can effectively shorten production cycle, improve production efficiency, decrease manufacturing cost, reduce the input of heat treatment equipment and personnel, as well as the pollution from heat treatment emissions to the environment, has prominent economic value and social benefits . In the grinding process, on the surface layer of the part may occur cracks or micro cracks if the grinding parameters are not chosen properly. When the temperature of grinding zone exceeds a certain threshold, the residual stress on the surface of the part may exceed the strength limit of the material, resulting in grinding cracks at the instantaneous cooling of the grinding liquid [1].

\section{Acoustic emission testing of grinding crack}

The appearance of grinding cracks in the surface layer has a great influence on the performance of the part in the grinding process, so online or scene testing has profound significance for timely detect crack generation or cracks. This paper adopts acoustic emission to test the grinding crack of parts.

Acoustic emission refers to the phenomenon that local material emits transient elastic wave due to the rapid release of energy. Acoustic emission testing is a very effective method to test the trend change of material under stress. The basic principle of acoustic emission testing is shown in figure 1[2]:

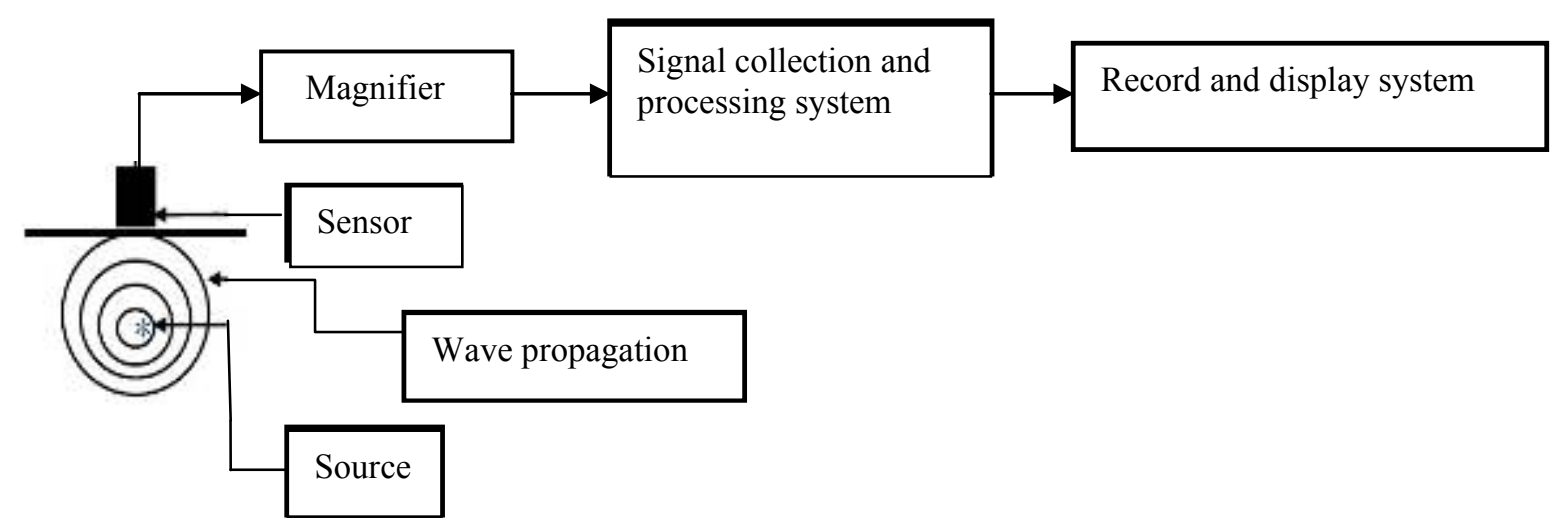

Figure 1. Acoustic emission testing principle diagram 
The elastic waves emitted from the acoustic emission source propagate in the material, finally get to the surface of the material, causing surface vibration signal that can be detected by acoustic emission sensor, as well as these sensors convert the mechanical vibration of the material into electrical signal and then magnify them with magnifier . Signals are collected, stored and processed in real-time by the signal collection cards and the corresponding collection software. Changes of internal stress in the solid material can produce acoustic emission signal, and there are many factors may cause internal stress changes in the process of material processing, such as dislocation movement, twinning, crack initiation and expansion, fracture, thermal expansion and cold contraction, load change ,etc. Research shows that many acoustic emission source produce transient elastic wave which can be received by taking advantage of special electronic instruments after a series of reflections and refraction to the surface of the material, then these electrical signals are converted into digital signals by a series of means to analyze them for useful information. Acoustic emission is a dynamic nondestructive testing method, which can monitor the object in real-time and high detection sensitivity. Moreover ,acoustic emission detection is not limited by material for almost all material have acoustic emission characteristic, and the size , geometry, working environment and other factors of the object have no effect on the testing[3,4].

For grinding crack detection, we choose digital acoustic emission detector of Beijing soundwel SAEU2S to collect signals, by using the signal analysis function of MATLB wavelet packet to find the singularity in the signal to determine the position of the $\operatorname{crack}[5]$.

\section{Acoustic emission signal analysis of grinding crack}

The main idea of denoising based on wavelet packet transform is to use multiscale characteristic of wavelet

a) Normal signal decomposition

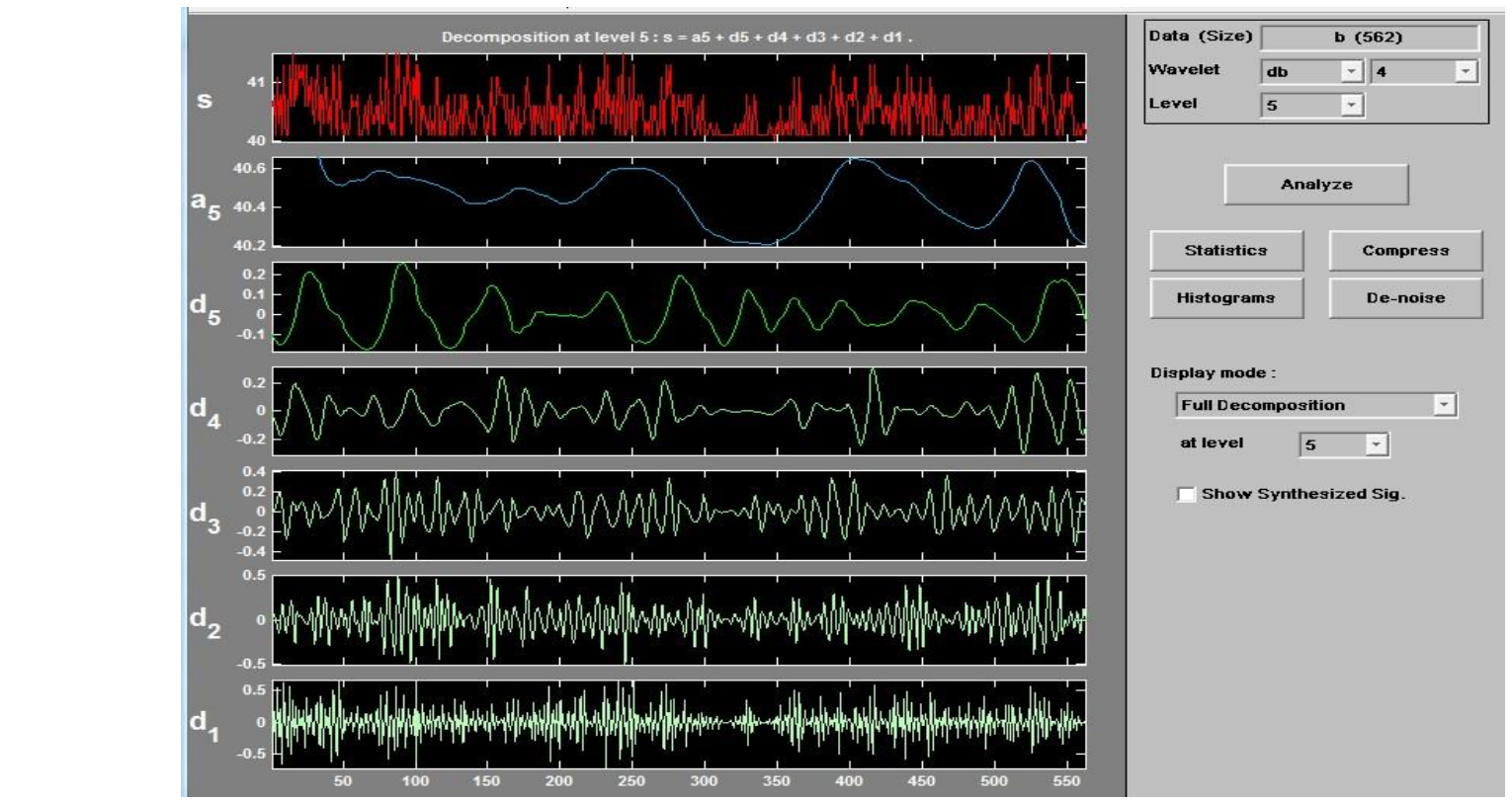

analysis. Firstly, wavelet transform is performed on noisy images. Secondly, the wavelet coefficients are processed by thresholding to get new wavelet coefficients. Lastly, the wavelet coefficients are processed with inverse transformation to get denoised images, realizing the recovery of the images. At present, many new image denoising algorithms have been developed by combining wavelet transform with traditional image denoising methods, they absorb advantages of both, and thus acquire a bright future for improving the denoising effect.

\section{Acoustic emission acquire signal}

The acoustic emission signal is collected by selecting those parts with cracks and no cracks on the surface after grinding hardening, then signal data is exported to MATLAB software to denoise the above signals by using MATLAB wavelet packet. Invoking the wavelet analysis toolbox, as shown in figure 2 :

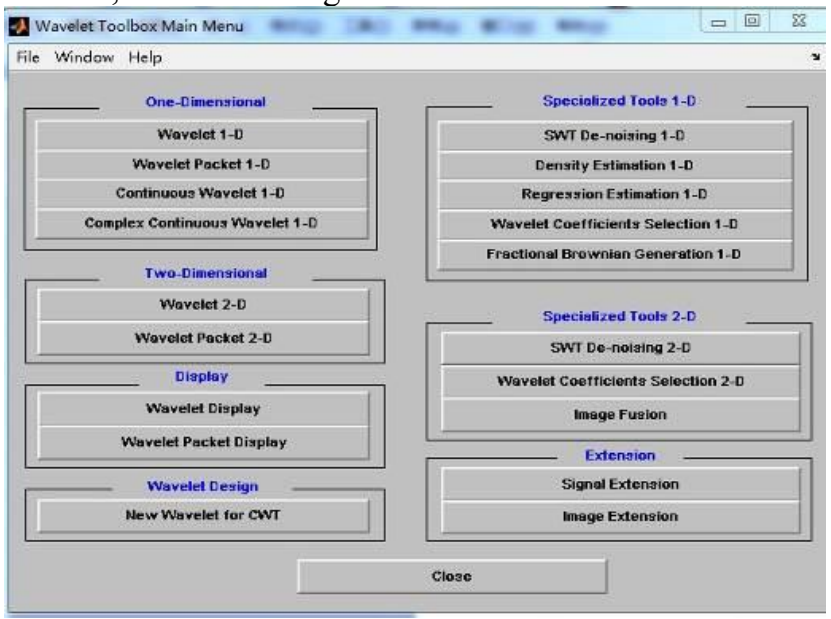

Figure 2. Wavelet packet panel

Selecting wavelet1-D,then import signal to be denoised, And wavelet is decomposed into a wave with 5 layers of $\mathrm{db} 4$ as wavelet base, as shown in figure 3:

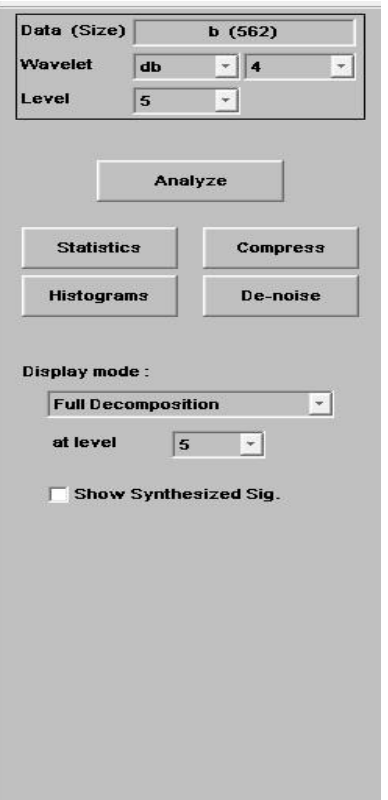




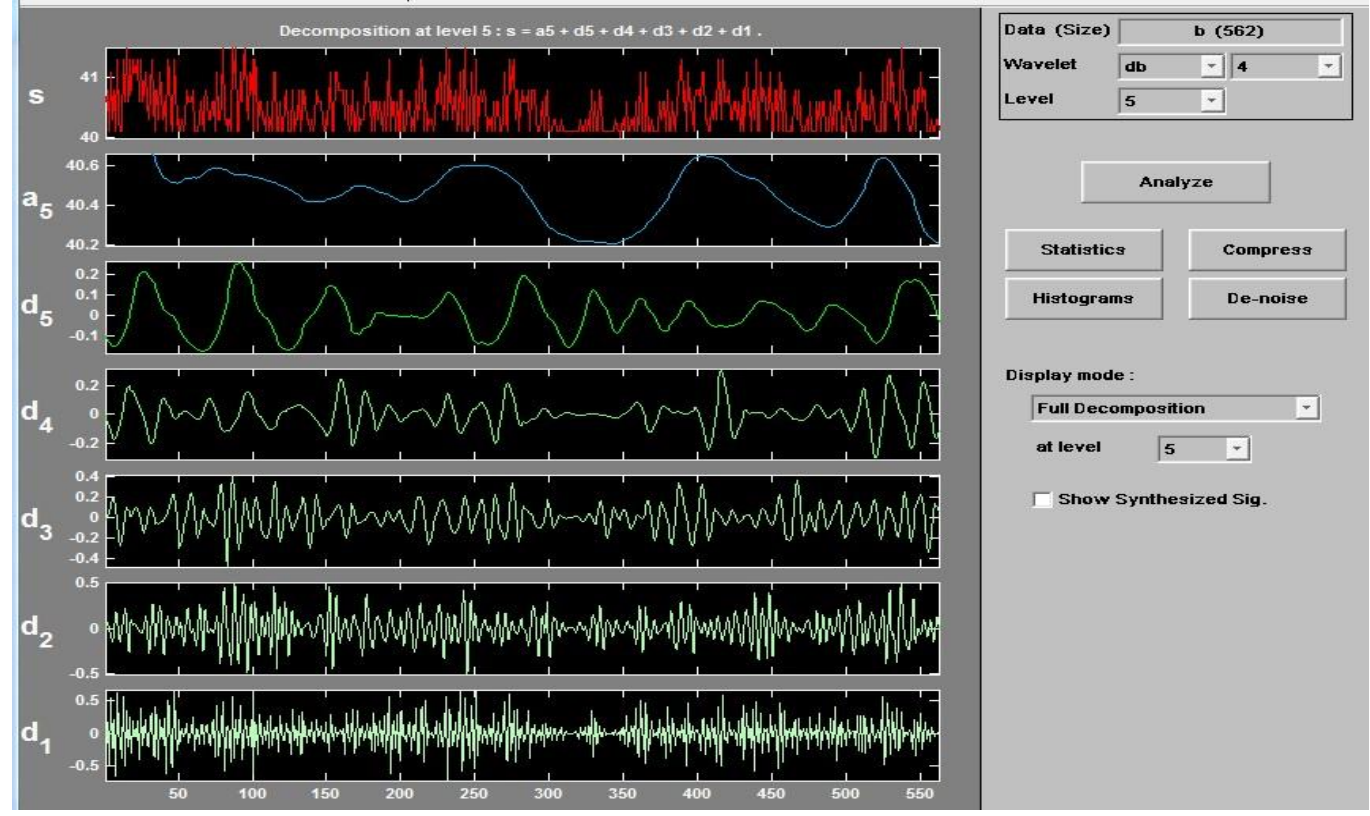

b) Signal decomposition of crack part

Figure 3. Decomposition of normal signal and cracked signal

Opening the denoised image and dividing it into 5 layers of

$\mathrm{db} 4$ wavelet to analyze it, as shown in figure 4 :
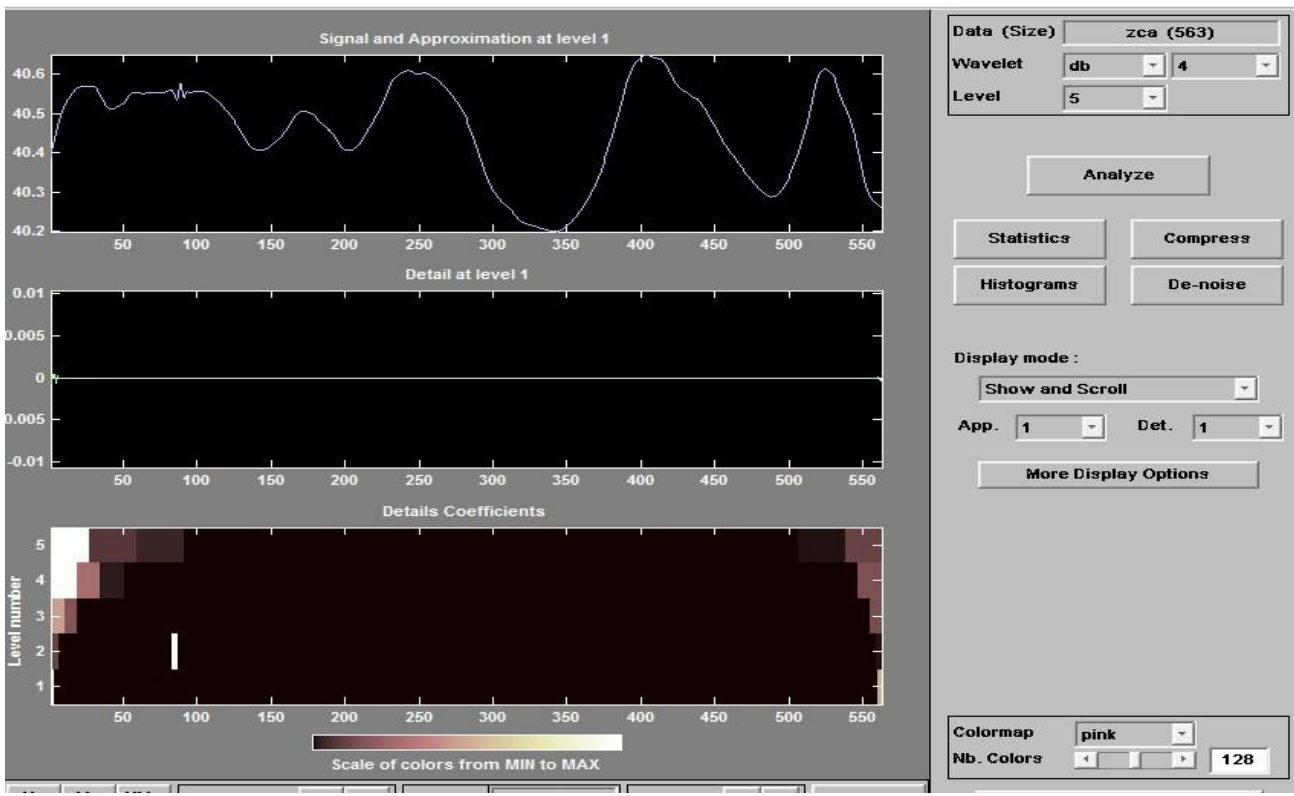

a) Denoising signal and wavelet transform diagram of normal signal 


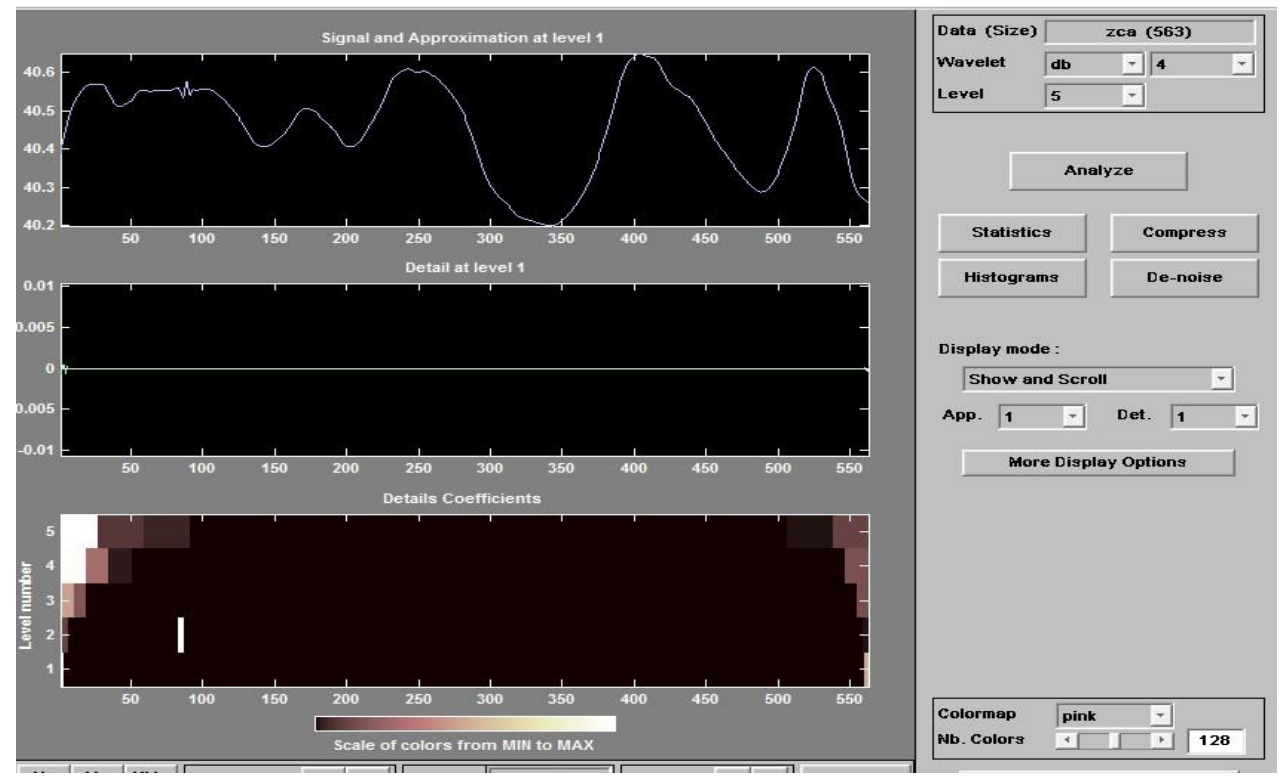

b) Denoising signal and wavelet transform diagram of cracked signal

Figure 4. Denoising signal and wavelet transform

The coefficients are present in the $b$ diagram from the first to the fifth floors and the wavelet coefficients have an inverted cone area at $X=+86.694$ and $X=+391.62$, which can be seen clearly in the diagram of the continuous wavelet transform coefficients. As a result, it is inferred that there is a mutation point in this area, so it can be concluded that crack defects exist in this region when grinding parts do not exist other defects or other defects have been detected.

\section{Conclusion}

The crack defects on the surface of the parts can be detected directly by comparing the wavelet data on the surface of normal parts and those parts with cracks on the surface after grinding hardening. Further, the location and size of cracks can be analyzed to provide technical support for active control surface quality of grinding hardened, development of integrated manufacturing by grind-hardening technology and Engineering application of automation technology.

\section{References}

1. Cheng wei, Wang guicheng. Automatic Control Technology of Grinding Temperature in Grinding Hardening Process. Advanced Materials Research, Vol.381:48-51.

2. LONG Xiao-jiang. Acoustic emission monitoring and evaluation for rolled steel damage under different tensile rates $[\mathrm{J}]$. Journal of Vibration and Shock, 2017, 36(7):219-225

3. LUO Zhi-gao, et al. State identification of a punching die based on characteristic analysis of acoustic emission signal $[\mathrm{J}]$. Journal of Vibration and Shock, 2009, 28(3):186-190.

4. SHEN Gong-tian, et al. Parameter analysis of acoustic emission signals $[\mathrm{J}]$. Nondestructive Testing(China), 2002, 24(2): 72-77.

5. WANG Wen-you. Acoustic Emission Testing Technique in Leak Detection of Pressure Pipeline[ $\mathrm{J}]$. Nondestructive Testing (China), 2016, 38(2): 22-24. 